\title{
Algas marinas bentónicas de la Isla Gorgona, costa pacífica colombiana
}

\author{
Mónica Murillo Muñoz ${ }^{1} \&$ Enrique Javier Peña Salamanca ${ }^{1 *}$ \\ 1. Facultad de Ciencias, Departamento de Biología, Universidad del Valle, Cali, Colombia; monicacheo@yahoo.com, \\ enrique.pena@correounivalle.edu.co
}

Recibido 18-X-2013. Corregido 20-XI-2013. Aceptado 19-XII-2013.

\begin{abstract}
Benthic marine algae of Gorgona island, Colombian Pacific coast. The knowledge of the biodiversity of Gorgona, a continental island in Colombia, is very limited in the case of algae. We present an updated list of the benthic marine algae of Gorgona Island, associated with different marine environments and type of substrates, such as coral reefs, rocks, and soft bottoms. Field samplings were taken between October 2010 and June 2011. We estimated algal cover, species composition and algal diversity for each reef environment using $0.25 \mathrm{~m}^{2}$ quadrats. Ecological and environmental aspects are also included. A total of 43 species of algae for the three types of habitats are documented: 24 Rhodophyta, 12 Clorophyta, six Ochrophyta and one species of Cyanobacteria, and $55 \%$ of the algal composition corresponded to red algae. Rev. Biol. Trop. 62 (Suppl. 1): 27-41. Epub 2014 February 01.
\end{abstract}

Key words: Chlorophyta, Ochrophyta, Rhodophyta, Cyanophyceae, algal cover, Gorgona Island.

El conocimiento de la flora de macroalgas marinas en el Pacífico Oriental Tropical es muy limitado. La flora algal del corredor marino de esta región, integrado por las islas de Coiba e Isla del Coco en Panamá, Isla Gorgona y Malpelo en Colombia, y la Reserva Marina de Galápagos en Ecuador, ha sido poco estudiado (Fernández, 2008; Fernández, Riosmena, Wysor, Tejada \& Cortés, 2011). Los estudios reportados en la región se han enfocado principalmente a listados taxonómicos. Los primeros trabajos se inician con la Expedición de la Allan Hancock, donde se reporta un total de 47 especies, de las cuales solamente cinco pertenecen a la división Chlorophyta (Taylor, 1945). Posteriormente, Dawson (1961) realiza colectas a lo largo de la costa pacífica desde Alaska hasta Galápagos incluyendo registros para la Isla Gorgona.

Los reportes de algas para la Isla Gorgona se inician con los trabajos de Lemoine (1929), quien registró nuevas especies de algas calcáreas coralináceas. Taylor (1945) realizó adiciones significativas a la flora algal de la isla, en el marco de la expedición Allan Hancock Foundation, la cual contribuyo al conocimiento de la biodiversidad de los ecosistemas marinos del Pacífico. Schnetter \& Bula (1982) elaboraron el compendio más completo hasta la fecha de la flora de algas marinas bénticas del Pacífico colombiano, complementando significativamente el estudio de la diversidad de algas para Gorgona. Bula (1995), publicó nuevos registros de macroalgas para la isla, proponiendo algunas hipótesis para la baja diversidad y biomasa de macroalgas.

En comparación con el litoral Caribe colombiano, la flora marina del Pacífico es significativamente menos diversa. Díaz \& Díaz (2003) reportaron un total de 565 especies para el Caribe colombiano. En contraste, Peña \& Palacios (1988) registraron un total de 133 especies para la zona continental del litoral pacífico colombiano, demostrando que la composición de algas de la Isla Gorgona contribuye con el $78 \%$ de biodiversidad 
para la región. Las posibles razones de la baja diversidad de algas en el Pacífico están relacionadas con factores como la pluviosidad, la salinidad, la turbidez en la columna del agua, y las condiciones desfavorables del sustrato (Schnetter \& Bula, 1982; Peña, 1998; Peña, 2008). Sin embargo, se requiere un mayor esfuerzo de muestreo para mejorar los registros ficológicos de la isla, especialmente en las zonas de mayor profundidad, donde las expediciones marinas no han realizado esfuerzos mayores.

Particularmente, la Isla Gorgona se encuentra en una situación especial, donde está muy poco influenciada por los derrames de agua dulce continentales, lo que permite la presencia de un número mayor de especies, comparada con la composición de la flora alga en la zona costera continental (Bula, 1988; Peña, Palacios \& Ospina, 2005). Adicionalmente, la presencia de formaciones coralinas en la isla, contribuye a la disponibilidad de un sustrato adecuado para la fijación de los talos. Por otro lado, la importancia de las macroalgas en el arrecife está asociada con su función en la formación de la estructura arrecifal, por cuanto pueden contribuir hasta en un $60 \%$ de la producción de carbonatos del ecosistema (Hughes \& Tanner, 2000).

El propósito de este trabajo fue actualizar el inventario de la comunidad de algas bénticas en la Isla Gorgona, incluyendo el análisis de su abundancia, cobertura y estructura relacionadas en dos tipos de ambientes marinos, fondos blandos someros y plataforma arrecifal.

\section{MATERIALES Y MÉTODOS}

Sitio de estudio: Isla Gorgona ( $2^{\circ} 55^{\prime} 45^{\prime \prime}$ $3^{\circ} 00^{\prime} 55^{\prime}$ ' N y $78^{\circ} 09^{\prime}-78^{\circ} 14^{\prime} 30^{\prime}$ ' W), constituye el territorio insular más extenso $\left(13,2 \mathrm{~km}^{2}\right)$ sobre la plataforma continental del Pacífico colombiano, y está ubicada a $30 \mathrm{~km}$ del continente. La isla se caracteriza por poseer una alta diversidad biológica tanto en sus hábitats terrestres como marinos, características que contribuyeron a reconocerla como un área importante del corredor marino en el Pacífico oriental tropical, razón por la cual la isla y un área marina de $610 \mathrm{~km}^{2}$ fueron declaradas Parque Natural Nacional en 1984. En la zona de influencia costera de la isla, se encuentran dos de los arrecifes coralinos más desarrollados y diversos del Pacífico oriental tropical, y el más grande del Pacífico Colombiano (Giraldo, Rodríguez \& Zapata, 2008). En este parque se encuentra una variedad de ambientes marinos y terrestres tales como los arrecifes coralinos, acantilados, playas coralinas, arenosas y rocosas, manglares y bosques húmedos, que en conjunto constituyen escenarios vitales para albergar una notable diversidad de especies de fauna y flora (Vásquez \& Serrano, 2009).

Recolección de muestras: Se recolectaron muestras en La Azufrada, Playa Blanca, La Ventana, La Camaronera, Playa Palmeras y El Muelle, localidades con sustratos coralinos y plataformas someras de fondos blandos. En cada sitio de muestreo se realizó un registro de las especies observadas y se evaluó la estructura de la comunidad de macroalgas de acuerdo a su composición y abundancia, medida en términos de porcentaje de cobertura en cuadrantes de $0.25 \mathrm{~m}^{2}$. Para este propósito, se trazaron dos trayectos lineales fijos de $100 \mathrm{~m}$, ubicados de forma paralela a la línea de costa, delimitando sobre cada trayecto 10 cuadrantes de muestreo. El porcentaje de cobertura algal se definió como el cociente entre el número de subcuadrantes ocupados por cada morfotipo, respecto del número total observado.

Las muestras fueron preservadas en húmedo, colocándolas en frascos plásticos con una solución de agua marina-formol al 4\%. Para las especies que presentaron estructuras reproductivas, se adicionó $1 \mathrm{~mL}$ de solución glicerina formalizada para su posterior identificación y trabajo morfológico. Algunas de las algas calcáreas colectadas se preservaron en seco, empacándolas en bolsas sellables de polietileno. Los ejemplares fueron incluidos en el Herbario CUVC de la Universidad del Valle.

Procedimientos de laboratorio: En el Laboratorio de Fisiología Vegetal de la 
Universidad del Valle, se realizó la confirmación de la identificación taxonómica siguiendo a Schnetter \& Bula (1982), Verheij \& Woelkerling (1992), Woelkerling \& Campbell (1992), Riosmena, Woelkerling \& Foster (1999), Harvey, Woelkerling \& Millar (2002), Riosmena (2007), y Amado et al., (2010). Se efectuaron cortes histológicos y observaciones en microscopio óptico de alta resolución, realizando un registro fotográfico para cada morfotipo.

\section{RESULTADOS}

Se identificaron 43 especies de algas asociadas a los ambientes coralinos y plataformas arenosas someras de isla Gorgona durante el presente estudio (Cuadro 1). El grupo Rhodophyta representó el $55.8 \%$ de la flora algal de la isla, seguida por Chlorophyta y Heterokontophyta con $27,9 \%$ y $14 \%$ respectivamente (Fig. 1). Los principales sustratos para el desarrollo de talos de la flora algal de la isla Gorgona corresponde a los arrecifes coralinos, los sustratos rocosos y las plataformas arenosas someras (Fig. 2). Las estaciones de muestreo con presencia de sustratos coralinos, registraron los mayores porcentajes de cobertura algal (87\%) mientras que los ambientes someros de plataformas arenosas de la isla

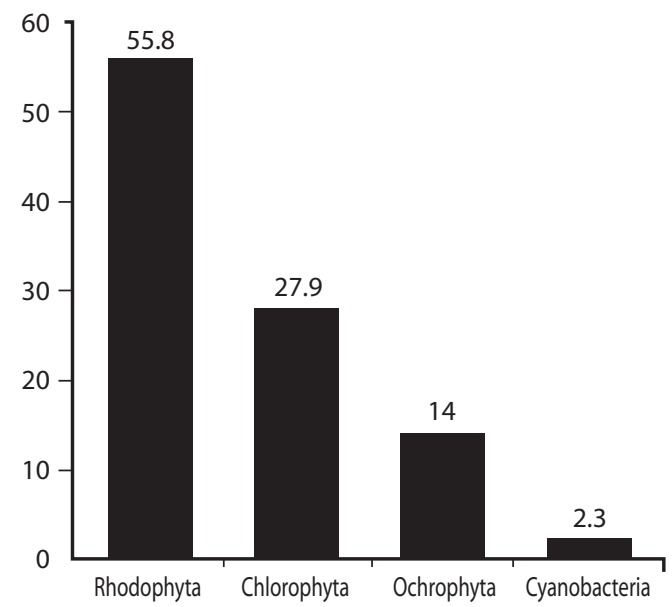

Fig. 1. Composición de la flora algológica en la Isla Gorgona.

Fig. 1. Algal flora composition of Gorgona Island. presentaron el menor porcentaje de cobertura (13\%) (Fig. 3). En el Cuadro 2 se relacionan los índices de diversidad establecido para cada uno de los ambientes.

Asociado al sustrato coralino de la estación La Azufrada, la especie con mayor cobertura fue el alga roja Sporolithon sp. (Fig. 4A
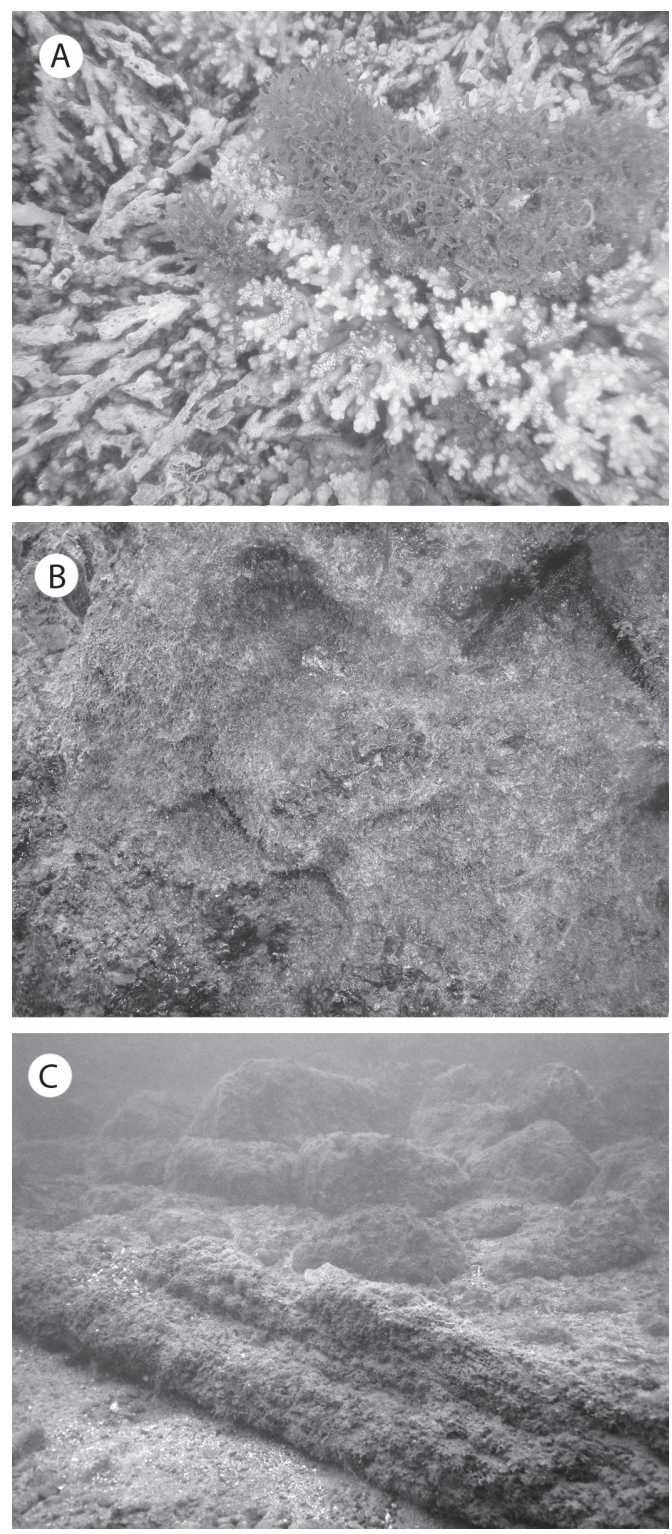

Fig. 2. Sustrato coralino (A, B) y fondos blandos (C) en isla Gorgona.

Fig. 2. Coral substrates (A, B) and soft-bottom environments (C) in Gorgona Island. 
CUADRO 1

Listado de las especies de algas bénticas asociadas a ambientes coralinos y plataformas marinas someras en la Isla Gorgona.

TABLE 1

Species composition of benthic algae from Gorgona island associated with coral and shallow soft-bottom environments.

\begin{tabular}{|c|c|c|c|}
\hline Phylum & Orden & Familia & Taxón \\
\hline Cyanobacteria & Oscillatoriales & Oscillatoriaceae & Phormidium sp. \\
\hline \multirow[t]{11}{*}{ Chlorophyta } & Dasycladales & Polyphysaceae & Acetabularia schenckii \\
\hline & & & Polyphysa clavata \\
\hline & Bryopsidales & Udoteaceae & Boodleopsis verticillata \\
\hline & & Bryopsidaceae & $\begin{array}{l}\text { Bryopsis galapagensis } \\
\text { Bryopsis } \mathrm{sp} .\end{array}$ \\
\hline & & Derbesiaceae & Derbesia attenuata \\
\hline & & Caulerpaceae & Caulerpa racemosa \\
\hline & & & Caulerpa peltata \\
\hline & & & Caulerpa macrophysa \\
\hline & Cladophorales & Cladophoraceae & Chaetomorpha califórnica \\
\hline & & & Cladophora albida \\
\hline & Siphonocladales & Boodleaceae & Cladophoropsis adhaerens \\
\hline \multirow[t]{6}{*}{ Ochrophyta } & Dictyotales & Dictyotaceae & Dictyota humifusa \\
\hline & & & Lobophora variegata \\
\hline & & & Padina crispata \\
\hline & & & Padina mexicana \\
\hline & Ectocarpales & Scytosiphonaceae & Colpomenia sinuosa \\
\hline & Sphacelariales & Sphacelariaceae & Sphacela riarigidula \\
\hline \multirow[t]{24}{*}{ Rhodophyta } & Corallinales & Corallinaceae & Amphiroa crosslandii \\
\hline & & & Spongites decipiens \\
\hline & & & Jania capillacea \\
\hline & & & Jania ungulata \\
\hline & & & Lithophyllum pallescens \\
\hline & & & Lithophyllum sp. \\
\hline & & & Lithothamnium sp. \\
\hline & & & Neogoniolithon sp. \\
\hline & & & Spongites sp. \\
\hline & & Hapalidiaceae & Mesophyllum crassiusculum \\
\hline & & & Mesophylum sp. \\
\hline & Ceramiales & Callithamniaceae & Callithamnion sp. \\
\hline & & Ceramiaceae & Ceramium procumbens \\
\hline & & Dasyaceae & Heterosiphonia sp. \\
\hline & & Rhodomelaceae & Bostrychia $\mathrm{sp}$. \\
\hline & & & Polysiphonia flaccidissima \\
\hline & & & Polysiphonia scopulorum \\
\hline & Gelidiales & Gelidiaceae & Gelidium pusillum var. cylindricum \\
\hline & & & Gelidium sp. \\
\hline & Gigartinales & Cystocloniaceae & Hypnea pannosa \\
\hline & & & Hypnea valentiae \\
\hline & Peyssonneliales & Peyssonneliaceae & Peyssonnelia sp. \\
\hline & Rhodymeniales & Lomentariaceae & Gelidiopsis sp. \\
\hline & Sporolithales & Sporolithaceae & Sporolithon sp. \\
\hline
\end{tabular}




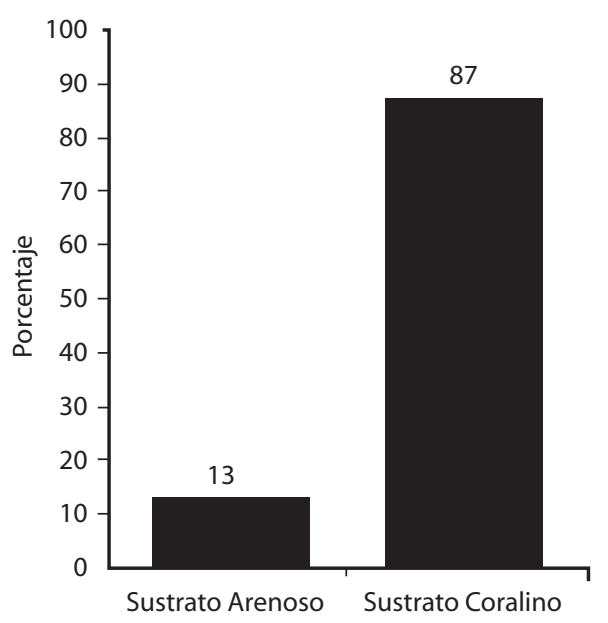

Fig. 3. Porcentaje de cobertura algal por sustrato.

Fig. 3. Porcentage of algal cover by substrate.

y 4B). En este tipo de ambientes fue abundante un tapete de algas (turf) compuesto por Lithophyllum pallescens (constituye la base del tapete), Callithamnion sp, Ceramium procumbens, Gelidium pusillum var. cylindricum, Polysiphonia scopulorum, Mesophyllum crassiusculum, Mesophyllum sp. (rodófitas); Acetabularia schenckii (clorófita), Bryopsis galapagensis, Cladophora albida, Derbesia attenuata, Chaetomorpha califórnica (clorófitas) y Sphacelaria rigidula (feófita). En la estación Playa Blanca las especies que presentaron mayor cobertura fueron las algas rojas Lithophylum sp, Gelidium pusillum sp, Sporolithon sp, Amphiroa crosslandii, e Hypnea valentiae y el alga verde Polyphysa clavata. En la estación La Ventana, el mayor porcentaje de cobertura estuvo conformado por otro tapete de algas de color café verdoso, el cual estuvo compuesto por Derbesia attenuata, Boodleopsis verticillata (Fig. 4C y 4D) y Cladophora albida; seguido igualmente por otra alga verde, Bryopsis sp. Asociado con el sustrato arenoso de la estación El Muelle las especies que presentaron mayor porcentaje de cobertura fueron Phormidium sp (cianofícea), y también se registró un tapete de algas de conformación similar al registrado en La Ventana.

A continuación se presenta una descripción del tipo, localidad, sinonimia, hábito y
CUADRO 2

Indices de diversidad en zonas de muestreo de la isla Gorgona.

TABLE 2

Diversity indexes of sampling areas in Gorgona Island.

\begin{tabular}{lcc} 
& Zona coralina & Plataforma arenosa \\
Dominancia D & 0.55 & 0.65 \\
Shannon H & 0.93 & 0.33 \\
Margalef & 1.55 & 1.18 \\
\hline
\end{tabular}

distribución geográfica de las diferentes especies de algas registradas en isla Gorgona durante el desarrollo de este trabajo.

\section{CYANOBACTERIA \\ Oscillatoriales \\ Phormidiaceae}

\section{Phormidium sp.}

Kützing ex Gomont (1892)

Hábito: Filamentos rara vez solitarios, usualmente cubriendo diferentes tipos sustratos acuáticos (suelo, rocas mojadas, barro, plantas acuáticas y madera, tanto en aguas estancadas como en corrientes), algunas especies se encuentran en el litoral marino. Varias habitan en localidades extremas (aguas termales, suelos de desierto, etc.), algunas de ellas participan en los procesos biolitogénicos y forman costras de travertino en biotopos de agua con piedra caliza.

\section{CHLOROPHYTA \\ Dasycladales \\ Polyphysaceae}

\section{Acetabularia schenckii}

K.Möbius (1889)

Sinónimos: Acicularia schenckii

(K.Möbius) Solms-Laubach 1895.

Localidad tipo:

Hábito: Sobre sustrato coralino, en la zona pre-arrecifal.

Distribución: Atlántico Occidental, Pacífico Oriental Tropical.

Estado fenológico: Reproductivo. 

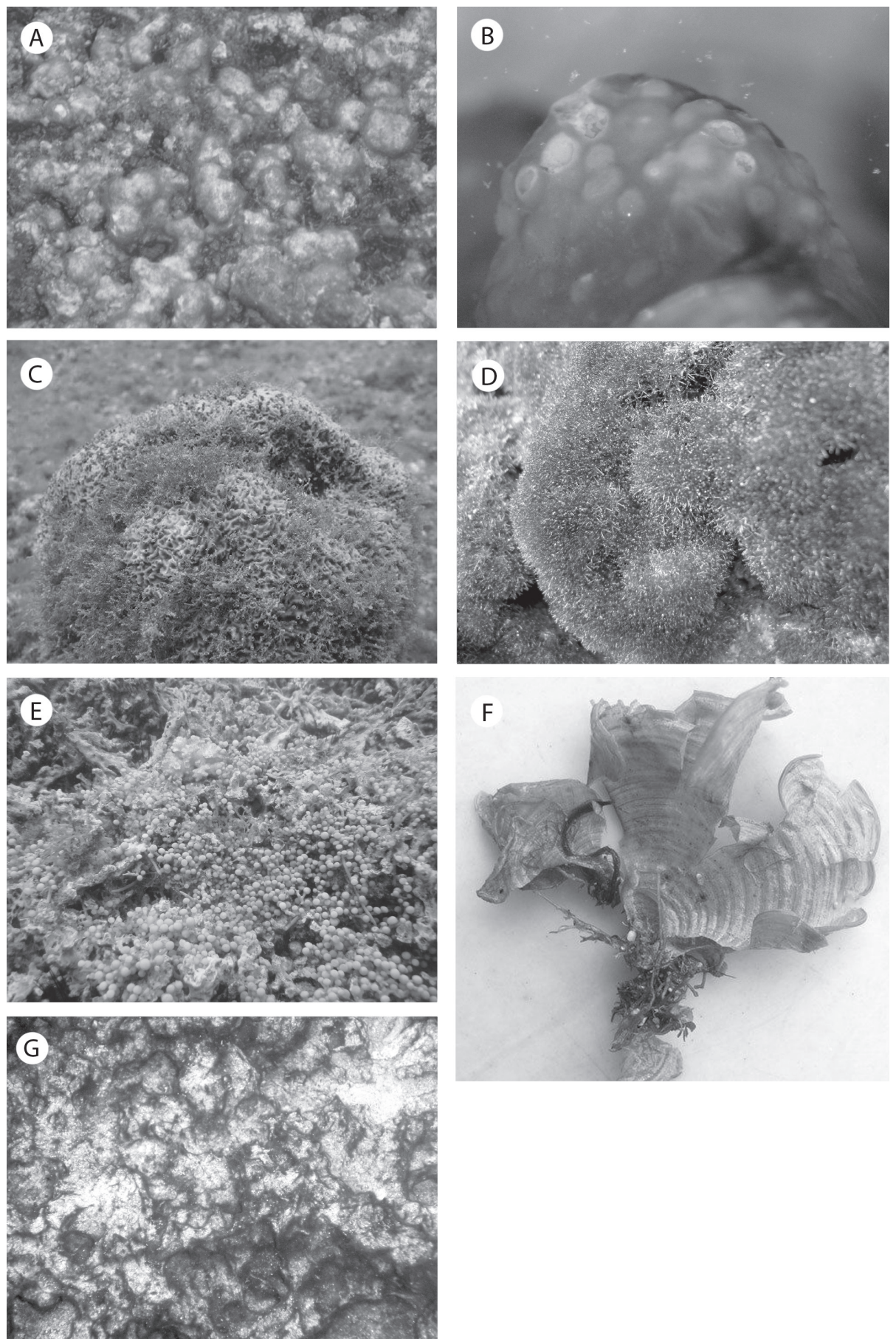

Fig. 4. Flora algologica representativa de la Isla Gorgona. (A) Sporolithon sp. (B) Propágulos de Sporolithon sp. (C) Derbesia attenuata. (D) Boodleopsis verticillata. (E) Caulerpa racemosa. (F) Padina crispata. (G) Phormidium sp.

Fig. 4. Main algal flora of Gorgona Island. (A) Sporolithon sp. (B) Propagules of Sporolithon sp. (C) Derbesia attenuata. (D) Boodleopsis verticillata. (E) Caulerpa racemosa. (F) Padina crispata. (G) Phormidium sp. 


\section{Polyphysa clavata}

(Yamada) Schnetter \& Bula (1982)

Localidad tipo: Tomari, Okinawa, Reino de Ryukyu, Japón (Silva, Basson \& Moe, 1996)

Hábito: Sobre sustrato coralino y rocoso, en planicie y talud arrecifal.

Distribución: Japón, Australia, Nueva Zelanda, Costa Rica, Colombia.

Estado fenológico: Reproductivo.

\section{Bryopsidales}

Udoteaceae

\section{Boodleopsis verticillata}

Dawson (1960)

Localidad tipo:

Hábito: Sobre sustrato coralino y rocoso, en zona pre-arrecifal, planicie y talud.

Distribución: Costa Rica, El Salvador, Panamá, Colombia, Filipinas.

Estado fenológico: Reproductivo.

\section{Bryopsidales \\ Bryopsidaceae \\ Bryopsis galapagensis}

Taylor (1945)

Localidad tipo: I. Wenman, Islas Galápagos, Ecuador (Taylor, 1945)

Hábito: Sobre sustrato coralino.

Distribución: El Salvador, México, Colombia, Islas Galápagos, Perú.

Estado fenológico: Reproductivo.

\section{Bryopsidales}

Derbesiaceae

\section{Derbesia attenuata}

E. Y. Dawson (1954)

Localidad tipo: Bahía de Nha Trang, Vietnam (Dawson, 1954)

Hábito: Abundante sobre fragmentos de coral entre $2-4 \mathrm{~m}$ de profundidad en el sublitoral.

Distribución: El Salvador, Panamá, Colombia, Filipinas, Vietnam, Australia, Nueva Zelanda.

Estado fenológico: Reproductivo.

\section{Bryopsidales \\ Caulerpaceae \\ Caulerpa racemosa}

(Forsskål) J. Agardh (1873)

Localidad tipo: Suez, Egipto (Silva, Basson \& Moe, 1996: 832)

Sinónimos: Fucus racemosus Forsskål (1775)

Hábito: Sobre sustrato rocoso. Crece formando cojinetes de aproximadamente $20 \mathrm{~cm}$ de diámetro y se encuentra con frecuencia desde la parte inferior de la zona intermareal hasta $5 \mathrm{~m}$ por debajo de la línea de baja mar.

Distribución: Cosmopolita, con carácter invasor a latitudes templadas.

Estado fenológico: Reproductivo.

\section{Caulerpa peltata}

J. V. Lamouroux (1809)

Localidad tipo: Antillas, Indias Occidentales (Silva, Basson \& Moe, 1996: 828)

Sinónimos: Ahnfeldtia peltata (J. V. Lamouroux) Trevisan, Chauvinia peltata (J. V. Lamouroux) Kützing (1849), Caulerpa racemosa var. peltata (J.V.Lamouroux) Eubank (1944)

Hábito: En pocetas de marea por encima de la línea de bajamar.

Distribución: Zonas tropicales

Estado fenológico: Reproductivo.

\section{Caulerpa macrophysa}

(Sonder ex Kützing) G. Murray (1887)

Localidad tipo: Centroamérica (Silva, Basson \& Moe, 1996: 837)

Sinónimo: Caulerpa racemosa var. macrophysa (Sonder ex Kützing) W. R. Taylor (1928)

Hábito: Sobre coral muerto.

Distribución: Zonas tropicales

Estado fenológico: Reproductivo.

\section{Cladophorales \\ Cladophoraceae \\ Chaetomorpha californica}

F. S. Collins in F. S. Collins,

Holden \& Setchell (1900)

Hábito: En la zona intermareal, sobre sustrato rocoso. 
Distribución: Pacífico Occidental, Colombia (costa pacífica)

Estado fenológico: Reproductivo.

\section{Cladophora albida}

(Nees) Kutzing (1843)

Localidad tipo: Selsey, Inglaterra (Womersley, 1984: 206)

Sinónimos: Annulina albida Nees (1820)

Hábito: En la zona intermareal, sobre sustrato rocoso.

Distribución: Cosmopolita.

Estado fenológico: Reproductivo.

\section{Siphonocladales \\ Boodleaceae \\ Cladophoropsis adhaerens \\ Gilbert (1962)}

Localidad tipo: Entre Natatorium y Elks Club, Waikiki, Honolulu, Oahu, Hawaii (Leliaert \& Coppejans, 2006)

Hábito: Talos erectos de hasta $2 \mathrm{~cm}$ de alto, formando tapetes fuertemente adheridos a sustrato rocoso.

Distribución: Islas de Hawái, Colombia.

Estado fenológico: Reproductivo.

\section{HETEROKONTOPHYTA Dictyotales Dictyotaceae Dictyota humifusa}

Hörnig, Schnetter \& Coppejans (1992)

Localidad tipo: Punta Chengue, Santa Marta, Departamento del Magdalena, Colombia (Silva, Basson \& Moe, 1996: 594)

Hábito: Se adhiere fuertemente a cualquier sustrato firme, en hábitats poco profundo y protegido.

Distribución: Pacífico colombiano, mar Caribe, Islas Canarias, océano Índico, pacífico occidental.

Estado fenológico: Reproductivo.

\section{Lobophora variegata}

(J. V. Lamouroux) Womersley ex E. C. Oliveira (1977)

Localidad tipo: Antillas, Indias Occidentales (Silva, Basson \& Moe, 1996: 598)
Sinónimos: Dictyota variegata J.V.Lamouroux (1809), Zonaria variegata (J.V.Lamouroux) C.Agardh (1817), Gymnosorus variegatus (J.V.Lamouroux) J.Agardh (1894), Pocockiella variegata (J.V.Lamouroux) Papenfuss (1943)

Hábito: Sobre sustrato rocoso, entre 1-9m de profundidad en el sublitoral.

Distribución: zonas tropicales.

Estado fenológico: Reproductivo.

\section{Padina crispata}

Thivy (1945)

(Dictyotales, Dictyotaceae)

Localidad tipo: Golfo Dulce, Costa Rica (Taylor, 1945)

Hábito: Sobre sustrato coralino, zona pre-arrecifal.

Distribución: México, Baja California, Costa Rica, Panamá, Colombia.

Estado fenológico: Reproductivo.

\section{Padina mexicana}

Dawson (1944)

Localidad tipo: Turners Island reef, off Tiburon Island (Dawson, 1944)

Hábito: Sobre sustrato coralino, zona pre-arrecifal.

Sinónimo heterotípico: Padina crispata Thivy in Taylor, 1945:100

Distribución: Pacífico Oriental Tropical.

Estado fenológico: Reproductivo.

\section{Ectocarpales \\ Scytosiphonales \\ Colpomenia sinuosa}

(Mertens ex Roth) Derbès \& Solier (1851)

Localidad tipo: Cerca de Cádiz, España (Roth 1806)

Sinónimos: Ulva sinuosa Mertens ex Roth (1806), Encoelium sinuosum (Mertens ex Roth) C.Agardh (1820), Stilophora sinuosa (Mertens ex Roth) C.Agardh (1827), Asperococcus sinuosus (Mertens ex Roth) Bory de Saint-Vincent (1832), Asperococcus sinuosus (C.Agardh) Zanardini (1841), Hydroclathrus sinuosus (Mertens) ex Roth) Zanardini (1843) 
Hábito: Zona intermareal, frecuente sobre sustrato rocoso.

Distribución: Zonas tropicales, Mar Mediterráneo.

Estado fenológico: Reproductivo.

\section{Sphacelariales \\ Sphacelaria \\ Sphacelaria rigidula}

Kützing (1843)

Localidad tipo: Nuweiba, Sinaí, Mar Rojo (Lipkin \& Silva, 2002)

Sinónimos: Sphacelaria furcigera Kützing (1855), Sphacelaria variabilis Sauvageau (1901), Sphacelaria iridaeophytica Nagai (1932), Sphacelaria caespitosa Takamatsu (1943), Sphacelaria linearis Takamatsu (1943), Sphacelaria apicalis Takamatsu (1943), Sphacelaria expansa Noda (1969), Sphacelaria iwagaskensis Noda (1970)

Hábito: Sobre sustratos rocosos o duros, epífita de otras algas; zona intermareal hasta $3 \mathrm{~m}$ de profundidad.

Distribución: cosmopolita.

Estado fenológico: Reproductivo.

\section{RHODOPHYTA \\ Corallinales \\ Corallinaceae \\ Amphiroa crosslandii \\ M. Lemoine (1929)}

Localidad tipo: Isla Gorgona (Dawson, 1953)

Hábito: Zonas intermedias y profundas, sobre sustrato coralino.

Distribución: Pacífico Oriental Tropical.

Estado fenológico: Reproductivo.

\section{Spongites decipiens}

(Foslie) Y.M. Chamberlain

Localidad tipo: California, U.S.A. (San Pedro) (Silva, Basson \& Moe, 1996: 271)

Sinonimo: Hydrolithon decipiens (Foslie) W.Adey (1970)

Hábito: Sobre sustrato rocoso.

Descripción: Talos costrosos, firmemente fijos al sustrato.

Distribución: Estados Unidos, Colombia.

Estado fenológico: Reproductivo.

\section{Jania capillacea}

Harvey (1853)

Localidad tipo: Bahía Honda, Florida, U.S.A. (Yoshida, 1998)

Hábito: Sobre sustrato rocoso, en zonas profundas.

Distribución: Zonas tropicales.

Estado fenológico: Reproductivo.

\section{Jania ungulata}

(Yendo) Yendo (1905)

Localidad tipo: Japón: Provincia de Wakasa (Prefectura de Fukui) y Misaki, Prefectura de Kanagawa (Silva, Basson \& Moe, 1996).

Sinónimos: Corallina ungulata Yendo (1902)

Hábito: Sobre sustrato coralino, en planicie y talud arrecifal.

Distribución: Brasil, Pacífico Oriental Tropical, Mar Arábigo, Océano Índico, Pacífico Occidental.

Estado fenológico: Reproductivo.

\section{Lithophyllum pallescens}

(Foslie) Foslie (1900)

Localidad tipo: Isla Espíritu Santo, Bajo California Sur, México (Silva, Basson \& Moe, 1996).

Sinónimos: Lithothamnion pallescens Foslie (1895)

Hábito: Sobre sustrato coralino y rocoso, en áreas someras, intermedias y profundas; talos costrosos o también de vida libre (rodolitos).

Distribución: Filipinas, México, Panamá, Colombia, Australia.

Estado fenológico: Reproductivo.

\section{Corallinales \\ Hapalidaceae \\ Lithothamnion sp.}

Heydrich (1897)

Localidad tipo: Bahía de Western Port, Victoria (Wilks \& Woelkerling 1995

Hábito: Sobre sustrato coralino y rocoso, en talud arrecifal.

Distribución: Brasil, Colombia, Chile, Corea, Australia, Nueva Zelanda.

Estado fenológico: Vegetativo. 
Neogoniolithon sp.

Setchell \& Mason (1943)

Localidad tipo: El Tor, Península del Sinaí, Egipto (Silva, Basson \& Moe, 1996)

Hábito: Talo costroso o libre consistente en masas de protuberancias; sobre sustrato coralino y rocoso, en planicie y talud arrecifal;

Distribución: Caribe, Océano Índico y Pacífico Occidental.

Estado fenológico: Vegetativo.

\section{Spongites sp.}

Kützing (1841)

Localidad tipo: Mar Mediterráneo (Silva, Basson \& Moe, 1996).

Hábito: Sobre sustrato coralino.

Distribución: Mar Mediterráneo, Atlántico Oriental, Océano Índico, Océano Pacífico Oriental.

Estado fenológico: Vegetativo.

\section{Mesophyllum crassiusculum}

(Foslie) Lebednik (2004)

Localidad tipo: White's Point, cerca de San Pedro, California (Setchell \& Mason, 1943).

Sinónimos: Lithothamnion rugosum $f$. crassiusculum Foslie (1901), Lithothamnion crassiusculum (Foslie) Mason (1943)

Hábito: Sobre sustrato coralino.

Distribución: Baja California, México, Colombia, Costa Rica, Japón, Corea.

Estado fenológico: Reproductivo.

\section{Ceramiales}

Callithamniaceae

Callithamnion sp.

Lyngbye (1819)

Localidad tipo: Brighthelmston, Sussex, Inglaterra (Silva, Basson \& Moe, 1996).

Hábito: Sobre sustrato coralino.

Distribución: Atlántico Oriental, Mar Mediterráneo, Mar Caribe, Mar Báltico, Pacífico Occidental.

Estado fenológico: Vegetativo.

\section{Ceramiales \\ Ceramiaceae \\ Ceramium procumbens}

Setchell \& N. L. Gardner (1924)

Localidad tipo: Isla Partida, Baja California, Mexico (Silva, Basson \& Moe, 1996)

Sinónimos: Ceramiella procumbens (Setchell \& Gardner) Díaz-Piferrer (1969)

Hábito: Sobre sustrato coralino, en zona pre-arrecifal.

Distribución: Pacífico Oriental Tropical, Océano Índico, Pacífico Occidental.

Estado fenológico: Reproductivo.

Ceramiales
Dasyaceae
Heterosiphonia sp.
Montagne (1842)

Hábito: Sobre sustrato rocoso.

Distribución: Colombia, Chile, Australia, Nueva Zelanda, Antártida.

Estado fenológico: Vegetativo.

\section{Ceramiales \\ Rhodomelaceae \\ Bostrychia radicans \\ Montagne (1842)}

Localidad tipo: Selsey-Sussex (Hudson 1762)

Hábito: Sobre sustrato rocoso.

Distribución: Caribe, Atlántico Occidental, Pacífico Oriental, Pacífico Occidental.

Estado fenológico: Reproductivo.

\section{Polysiphonia flaccidissima}

Hollenberg (1942)

Localidad tipo: Laguna Beach, California (Silva, Basson \& Moe, 1996)

Hábito: Sobre sustrato coralino, en planicie y talud arrecifal.

Distribución: Bermudas, California, Carolina del Norte, Belice, Costa Rica, El Salvador, Panamá, Colombia, Perú, Chile, Islas Hawaianas.

Estado fenológico: Reproductivo. 


\section{Polysiphonia scopulorum}

Harvey (1855)

Localidad tipo: Isla Rottnest, Australia Occidental (Silva, Basson \& Moe, 1996)

Sinónimos: Vertebrata scopulorum (Harvey) Kuntze (1891), Lophosiphonia scopulorum (Harvey) Womersley (1950)

Hábito: Sobre sustrato coralino.

Distribución: Mar Mediterráneo, Islas Canarias, Isla Gough, Islas Cabo Verde, Madeira, Alaska, California, Mar Caribe, Brasil, Colombia, Océano Pacífico Occidental.

Estado fenológico: Reproductivo.

\section{Gelidiales}

Gelidiaceae

Gelidium pusillum var. cylindricum

Taylor (1945)

Localidad tipo: Bahía San Francisco, Esmeraldas, Ecuador (Taylor 1945)

Hábito: Sobre sustrato coralino, en planicie arrecifal.

Distribución: Colombia, Ecuador, China, Corea.

Estado fenológico: Reproductivo.

\section{Hypnea pannosa}

J.Agardh (1847)

(Gigartinales, Cystocloniaceae)

Localidad tipo: San Agustín (Oaxaca), México (South, 2004)

Sinónimos: Hypnea musciformis var. cornuta Harvey (1834)

Hábito: Sobre sustrato coralino, en planicie arrecifal.

Distribución: Golfo de California, Costa Rica, El Salvador, México, Panamá, Brasil, Pacífico Oriental Tropical, Océano Índico, Océano Pacífico Occidental.

Estado fenológico: Reproductivo.

\section{Gigartinales}

Cystocloniaceae

Hypnea valentiae

(Turner) Montagne (1841)

Localidad tipo: Mar Rojo (Dawson, 1961)
Sinónimos: Fucus valentiae Turner (1809), Hypnea musciformis var. valentiae (Turner) Harvey (1834)

Hábito: Sobre sustrato coralino, en planicie arrecifal.

Distribución: Mar Mediterráneo, Pacífico Oriental, Mar Caribe, Océano Índico, Mar Arábigo.

Estado fenológico: Reproductivo.

\section{Peyssonneliales}

Peyssonneliaceae

Peyssonnelia sp.

Decaisne (1841)

Localidad tipo: Mar Mediterráneo (Athanasiadis, 1996)

Hábito: Sobre sustrato coralino, en planicie arrecifal.

Distribución: mar Mediterráneo, México, Colombia, Brasil, China, Indonesia, Filipinas.

Estado fenológico: Vegetativo.

\section{Rhodymeniales \\ Lomentariaceae \\ Gelidiopsis sp.}

F. Schmitz (1895)

Localidad tipo: Madrás, India (Silva, Basson \& Moe, 1996)

Hábito: Sobre sustrato coralino.

Distribución: Pacífico Oriental Tropical, Pacífico Occidental, Mar Caribe, Océano Atlántico Oriental, Océano Índico.

Estado fenológico: Vegetativo.

\section{Sporolithales}

Sporolithaceae

Sporolithon sp.

Heydrich, (1897)

Localidad tipo: El Tor, Península del Sinaí, Egipto (Silva, Basson \& Moe, 1996)

Hábito: Sobre sustrato coralino y rocoso, en planicie y talud arrecifal.

Distribución: Mar Mediterráneo, Brasil, Colombia, Océano Índico, Indonesia, Pacífico Sur.

Estado fenológico: Vegetativo. 


\section{DISCUSIÓN}

Los resultados obtenidos en isla Gorgona mostraron la dominancia de las algas rojas -especialmente de tipo incrustante- concordando con lo reportado por Bula (1995). Las zonas que presentaron más número de especies fueron las correspondientes a sustratos coralinos (La Azufrada y Playa Blanca), las cuales se encuentran ubicadas en el extremo oriental, formando un ecosistema de alta importancia para la diversidad marina de la isla (Mann 2000, Zapata \& Vargas 2003). Las macroalgas son un componente primordial en las comunidades de arrecifes coralinos ya que cumplen una importante función ecológica y ambiental en las áreas donde estos se desarrollan (Dawes, 1991; McCook, 1996).

El alga roja calcárea del genero Sporolithon $s p$, correspondió al grupo de las algas calcáreas que se reportó en las seis estaciones de muestreo y en todos los tipos de ambientes ecológicos. La importancia del estudio de las coralináceas (algas rojas) radica en el papel que desempeñan en la formación (cementación y consolidación) de los arrecifes coralinos (Celis, Álvarez \& Bula, 1999). La estación con los porcentajes de cobertura de algas más altos fue La Azufrada, en la zona de la planicie arrecifal área que coincide con el porcentaje de coral muerto deteriorado más alto del arrecife (Zapata, Vargas \& Garzón, 2001, Zapata \& Vargas, 2003). Esta condición ha favorecido la colonización por algas en este sector (Peña et al., 2005), en contraste los porcentajes de cobertura en la planicie arrecifal de Playa Blanca fue mucho menor.

Callithamnion sp, Ceramium procumbens, Gelidium pusillum var. cylindricum, Polysiphonia scopulorum, Mesophyllum crassiusculum, Mesophyllum sp (Rodophyta); Acetabularia schenckii (Chlorophyta), Bryopsis galapagensis, Cladophora albida, Derbesia attenuata, Chaetomorpha californica (Chlorophyta) y Sphacelaria rigidula (Phaeophyta) conforman los tapetes algales, que registran los mayores porcentajes de cobertura en los tipos de sustratos estudiados, coralino (estación La Azufrada) y arenoso (estación El Muelle). Otro tipo de tapete de color café-verdoso fue encontrado sobre sustratos rocosos en la estación de $\mathrm{La}$ Ventana. Estas formaciones algales se caracterizan por presentar un crecimiento de tipo filamentoso (Sánchez \& Bolívar, 1997). Este tipo de crecimiento está asociado en parte, al control que ejercen los peces herbívoros, para formar "jardines algales" donde "las cultivan" cuidadosamente para su alimento (Zapata et al., 2001; Rodríguez, Navas, Reyes, Bejarano \& Garzón, 2007). Sin embargo otros autores como Sánchez \& Bolívar (1997), consideran que la agrupación de varias especies en forma de tapetes es una estrategia simbiótica que les permite depositar sedimentos para fijación del talo como sustrato y la acumulación materia orgánica.

Tanto en las zonas de sustrato coralino (La Azufrada y Playa Blanca) como en la de sustrato arenoso (El Muelle) se evidenció la presencia de la cianobacteria Phormidium sp. en forma de masa café-rojiza sobre la superficie de los corales blanqueados y en la columna de agua (Fig. 3G), con coberturas considerables en comparación con otras algas presentes en las mismas zonas. Su morfología es de tipo filamentosa formando hilos de $5 \mathrm{~cm}$ o más largos los cuales pueden ocupar grandes áreas de sustrato coralino. Las altas coberturas pueden llegar a tener efectos negativos sobre el crecimiento y desarrollo coralino (Obura, 2009). La mayor diversidad de especies se registró en las estaciones de sustratos coralinos en comparación con la diversidad de las zonas correspondientes a los ambientes areno-fangosos.

El análisis de los resultados de la cobertura algal concluye que los mayores porcentajes de cobertura se presentaron en el arrecife de la Azufrada y Playa Blanca. La presencia de las formaciones de tapetes de algas puede estar asociada a una estrategia de la fauna íctica herbívora que favorece la distribución espacial agregada sobre la zona en el arrecife. Los ambientes coralinos de la isla albergan la mayor diversidad de algas, observaciones que son consistentes con la estructura de la diversidad de las comunidades algales reportadas para 
otras zonas insulares en el Pacífico Tropical Oriental. La alta diversidad y cobertura algal de estos ambientes coralinos contrasta con el resto de ambientes costeros del Pacífico colombiano donde se ha registrado bajos índices de diversidad (Peña, 2008). La isla Gorgona se convierte por lo tanto, en un enclave fitogeográfico importante en términos de su alta diversidad de la flora algológica, particularmente por sus variados biotopos de sustratos duros que permiten un mayor desarrollo de las macroalgas (Bula, 1995; Peña, 2008; Palacios, et al., 2012).

Desde el punto de vista de la composición florística algológica de la isla, el número de especies reportadas a la fecha representa $32.3 \%$ del total de 133 especies documentadas para el Pacífico colombiano. (Peña, Palacios \& Mejía, 1987; Peña \& Palacios, 1988; Peña 1998; Díaz \& Díaz, 2003; Álvarez, Pardo \& Trespalacios, 2007). El presente trabajo reporta un total de 17 nuevos registros, lo que contribuye a actualizar los inventarios florísticos de algas para el Pacífico oriental tropical. Estos nuevos registros ratifican la importancia fitogeográfica de la isla, por cuanto la composición florística insular contribuye significativamente a la biodiversidad para la región, dado que esta se encuentra en una situación especial, donde está muy poca influenciada por los derrames de agua dulce continentales (Bula, 1988; Peña et al., 2005). En el presente trabajo no se muestrearon ambientes rocosos de la isla, localidades que fueron estudiadas por Schnetter y Bula (1982), por cuanto se focalizaron las comunidades algales de ambientes arrecifales. Bula (1995), destacó igualmente, la presencia de formaciones coralinas en la isla para explicar la alta diversidad de macroalgas reportadas en su estudio, siendo este un factor a destacar al comparar con el resto de la línea costera del Pacífico colombiano.

\section{AGRADECIMIENTOS}

A Hernel Marín, por su apoyo en la estimación de los datos de cobertura y en la edición del manuscrito. Este estudio se desarrolló en el marco del proyecto "Evaluación del estado actual de los objetos de conservación faunísticos en isla Gorgona: una aproximación holística a la valoración ecológica de PNN Gorgona", financiado por la Universidad del Valle, la Fundación Squalus, el Fondo para la Acción Ambiental y la Niñez, Conservación Internacional Colombia y Parques Nacionales Naturales, amparado por el permiso de investigación PIBD-DTSO 011-10. A la estación científia Henry von Prahl del PNN Gorgona por el apoyo logístico durante las jornadas de campo.

\section{RESUMEN}

Se presenta el listado de las algas marinas bénticas del Parque Nacional Natural Isla Gorgona, el inventario corresponde a la flora algal asociada a los diferentes ambientes marinos de la isla, a saber: arrecifes coralinos y fondos blandos. El monitoreo de las comunidades de algas se llevó a cabo entre octubre de 2010 y mayo de 2011, se realizó una estimación de abundancia y cobertura para lo cual se establecieron transeptos paralelos a la línea de costa y se evaluó el porcentaje de cobertura en cuadrantes de $0.25 \mathrm{~m}^{2}$. Se reportó un total de 43 especies para los dos ambientes estudiados (coralino y fondos blandos), 24 Rhodophyta, 12 Clorophyta, 6 Ochrophyta y una Cyanobacteria. El grupo más representativo fue el perteneciente a la clase Rhodophyceae, que correspondió al 55.8\% de la flora inventariada. Los resultados de nuevos registros en la isla ratifican su importancia fitogeográfica, por cuanto la composición florística insular contribuye significativamente a la biodiversidad de la costa pacífica colombiana, destacándose la presencia de arrecifes coralinos como los principales ambientes marinos para el desarrollo de la flora algal de la región.

Palabras clave: Chlorophyta, Ochrophyta, Rhodophyta, Cyanophyceae, Isla Gorgona.

\section{REFERENCIAS}

Álvarez, R., Pardo, C. \& Trespalacios, A. (2007). Evaluación y utilización potencial de las macroalgas marinas del Caribe y el Pacífico de Colombia: estado actual de su conocimiento. Biosalud, 6: 113-129.

Amado, G. M., Maneveldt, G. W., Pereira, G. H., Manso, R. C. C., Bahia, R. G., Barros, M. B. \& Guimarães, S. M. P. B. (2010). Seaweed diversity associated with a Brazilian tropical rhodolith bed. Ciencias Marinas, 36: 371-391.

Bula, G. (1988). Cultivos y utilización comerciales de las macroalgas marinas. Revista Ingeniería Pesquera, 6: $1-57$. 
Bula, G. (1995). Macroalgas de la Isla de Gorgona (Pacífico Colombiano) con nuevos registros y una explicación de la baja diversidad y biomasa. La isla de Gorgona, nuevos estudios biológicos. Instituto de Ciencias Naturales-Museo de Historia Natural, Biblioteca José Jerónimo Triana. Santafé de Bogotá, Colombia.

Celis, A., Álvarez, R. \& Bula, G. (1999). Epifitismo y Epizoismo de Algas Coralináceas (Rhodophyta: Corallynaceae) en el Archipiélago del Rosario, Caribe Colombiano. Caribbean Journal of Science, 35: 296-303.

Dawes, C. J. (1991). Botánica Marina. Limusa. México.

Dawson, E. Y. (1961). A guide to the literature and distributions of Pacific benthic algae from Alaska to the Galapagos Islands. Pacific Sciencie, 15: 370-461.

Díaz, G. \& Díaz, M. (2003). Diversity of benthic marine algae of the Colombian Pacific. Biota Colombiana, 4: 203-246.

Fernández, C. (2008). Flora marina del Parque Nacional Isla del Coco, Costa Rica, Pacífico Tropical Oriental. Revista de Biología Tropical, 56: 57-69.

Fernández, C., Riosmena, R., Wysor, B., Tejada, O., \& Cortés, J. (2011). Checklist of the Pacific marine macroalgae of Central America. Botanica Marina, 54: 53-73.

Giraldo, A., Rodríguez, E. \& Zapata, F. (2008). Condiciones oceanográficas en isla Gorgona, Pacífico Oriental Tropical de Colombia. Latin American Journal of Aquatic Research, 36: 121-128.

Harvey, W., Woelkerling, J. \& Millar, A. J. K. (2002). The sporolithaceae (Coralinalles, Rhodophyta) in southeastern Australia: taxonomy and 18s rRNA phylogeny. Phycologia, 41: 207-227.

Hughes, T. P. \& Tanner, J. E. (2000). Recruitment failure, life histories and long-term decline of Caribbean corals. Ecology, 81: 2250-2263.

Lemoine, M. P. (1929). Les corallinacées de l'Archipel des Galapagos et du Golfe de Panama. Archives du Muséum National d'Histoire Naturelle, 4: 37-88.

Mann, H. (2000). Ecology of coastal waters: with implication for management. Blackwell Sciences, Massachusetts, USA.

McCook, L. J. (1996). Effects of herbivores and water quality on Sargassum distribution on the Central Great Barrier Reef: cross-shelf transplants. Marine Ecology Progress Series, 139: 179-192.
Obura, D. (2009). Coral Reef Resilience Assessment of the Nosy Hara Marine Protected Area, Northwest Madagascar. IUCN. Switzerland.

Palacios, M., Zapata, F., Peña, E. \& Murillo, M. (2012). Las formaciones coralinas del Parque Nacional Natural Gorgona. En Isla Gorgona: Paraíso de Biodiversidad y Ciencia.

Peña, E. J., Palacios, M. L., \& Mejía, A. (1987). Estudio fitosociológico de una zona intermareal en la Bahía de Málaga, costa pacífica colombiana. Revista Asociación Colombiana de Ciencias Biológicas, 4(1): $12-21$

Peña, E. J. (1998). Physiological ecology of mangrove associated macroalgae in a tropical estuary (Doctoral dissertation). University of South Carolina, USA.

Peña, E. J. \& Palacios, M. L. 1988. Macroalgas marinas bénticas asociadas al manglar de la Costa Pacífica colombiana. En Memorias VI Sem. Nal. y Tec. del Mar. Universidad Jorge Tadeo Lozano. Bogotá, Colombia.

Peña, E. J., Palacios, M. L. \& Ospina, N. (2005). Algas como indicadores ambientales. Universidad del Valle. Cali, Colombia.

Peña, E. J. (2008). Dinámica espacial y temporal de la biomasa algal asociada a las raíces de mangle en la Bahía de Buenaventura, costa pacífica de Colombia. Boletín de Investigaciones Marinas y Costeras, 37: 55-70.

Riosmena, R., Woelkerling, W. J. \& Foster, M. S. (1999). Taxonomic reassessment of rhodolith-forming species of Lithophyllum (Corallinales, Rhodophyta) in the Gulf of California, Mexico. Phycologia, 38: 401-417.

Riosmena, R. (2007). Guidelines to prepare the information from your specimens to assemble the species description that will be use for thesis publications. Programa de Investigación en Botánica Marina. México.

Rodríguez, A., Navas, C., Reyes, N., Bejarano. S. \& Garzón, J. (2007). Implementación del SIMAC en la isla Malpelo. Santuario de flora y fauna Malpelo: descubrimiento en marcha. Dimar. Santafé de Bogotá, Colombia.

Sánchez, P. \& Bolívar, F. (1997). Caracterización de comunidades algales epifitas en fuentes monumentales y su aplicación a la diagnosis del biodeterioro. Lirnnetica, 13: 31-46.

Schnetter, R. \& Bula, G. (1982). Algas marinas del litoral pacífico de Colombia, Clorophyceae, Phaeophyceae, Rhodophyceae. J. Cramer. Alemania. 
Taylor, W. R. (1945). Pacific marine algae of the Allan Hancock Expeditions to the Galapagos Islands. Allan Hancock Pacific Expeditions, 12: 1-528.

Vásquez, V. \& Serrano, M. (2009). Las áreas naturales protegidas de Colombia. Conservación internacionalColombia y fundación Biocolombia. Santafé de Bogotá, Colombia.

Verjeij, E. \& Woelkerling, W. J. (1992). The typification of nongeniculated corallines (Rhodophyta) involving siboga expedition collections. Blumea, 36: 273-291.

Woelkerling, W. J. \& Campbell, S. J. (1992). An account of southern Australian species of Lithophyllum
(Corallinaceae, Rhodophyta). Bulletin British Museum (Natural History) Botany, 22: 1-107.

Zapata, F. A., Vargas, B. \& Garzón, J. (2001). Salud y conservación de las comunidades coralinas. In L. M. Barrios \& M. López (Eds.). Gorgona Marina: Contribución al conocimiento de una isla única (pp. 41-50). Santa Marta, Colombia: INVEMAR.

Zapata, F. A. \& Vargas, B. (2003). Corals and coral reefs of the Pacific coast of Colombia. In J, Cortes. (Ed.), Latin America coral reefs (pp. 419-447). Amsterdam, Netherlands: Elsevier Science. 
